\title{
Article
}

\section{MWQOE: A user-centered context-aware model for evaluating the Mobile Web Quality of Experience}

Antoniou, Josephina and Allayiotis, Elias

Available at http://clok.uclan.ac.uk/19510/

Antoniou, Josephina ORCID: 0000-0003-0169-1299 and Allayiotis, Elias (2018) MWQoE: A user-centered context-aware model for evaluating the Mobile Web Quality of Experience. Proceedings of IEEE International Symposium on Personal, Indoor, and Mobile Radio Communications 2017.

It is advisable to refer to the publisher's version if you intend to cite from the work. http://dx.doi.org/10.1109/PIMRC.2017.8292534

For more information about UCLan's research in this area go to http://www.uclan.ac.uk/researchgroups/ and search for < name of research Group>.

For information about Research generally at UCLan please go to http://www.uclan.ac.uk/research/

All outputs in CLoK are protected by Intellectual Property Rights law, including Copyright law. Copyright, IPR and Moral Rights for the works on this site are retained by the individual authors and/or other copyright owners. Terms and conditions for use of this material are defined in the policies page.

\section{CLoK}

Central Lancashire online Knowledge www.clok.uclan.ac.uk

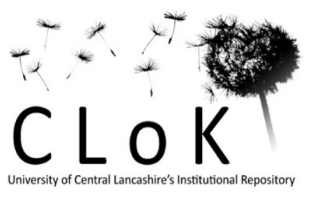




\section{MWQoE: A user-centered context-aware model for evaluating the Mobile Web Quality of Experience}

\author{
Josephina Antoniou \\ School of Sciences \\ University of Central Lancashire, \\ 12-14 University Av., Pyla, 7080 Larnaca, Cyprus. \\ Email: JAntoniou@uclan.ac.uk
}

\author{
Elias Allayiotis \\ School of Physical Sciences and Computing \\ University of Central Lancashire, \\ Preson, Lancashire, UK, PR12HE. \\ Email: EAllayiotis@uclan.ac.uk
}

\begin{abstract}
This article presents a human-system interaction modelling approach for quantifying Web Quality of Experience (Web QoE) for mobile devices. It builds on current QoE and Web QoE research, and by fusing together data that is available on modern mobile devices, constructs a novel Mobile Web QoE (MWQoE) model that is user-centered and context-aware. The MWQoE model uses Bayesian Networks and works under uncertainty, while the MWQoE metric uses Utility Theory and delivers a quantified characterization of MWQoE on a single scale in specific scenarios. The importance of MWQoE lies in the fact that online content and service providers need insights into their users in order to understand how the experience in using their products is perceived.
\end{abstract}

\section{INTRODUCTION}

Understanding what makes mobile users happy, i.e. which technology or service features are better received, can be quite important to content, service, and application providers, in order to, on the one hand, keep existing customers, and, on the other, attract new ones. The Quality of Experience (QoE) of technology/content online consumers is becoming an increasingly popular area of interest for both academia and industry. Online providers continuously strive to provide an improved user journey through their digital services by carefully crafting features and web session sequences that would be better received by their customers [1].

There is an ever-increasing shift towards simplifying and redesigning user interfaces for ease of use while at the same time web analytics' providers are becoming an absolute necessity for any service that needs insights into their user base.

This focus on QoE is increasing and becoming more fine-grained. Initial QoE studies dealt with aligning QoE with Quality of Service (QoS), by identifying relationships of quantitative network measures with qualitative user perception metrics, and linking these back into QoE definitions. Therefore, generic relationships between QoS and QoE have been observed such as the WQL hypothesis [2], which reveals a logarithmic relationship between QoS and QoE, and, the IQX hypothesis [3], which reveals an exponential relationship between the two. Most QoE research studies focus on multimedia scenarios such as video and voice transmissions and much less on Web QoE (WQoE) and Mobile Web QoE (MWQoE).

In order for online services or content delivery to be improved there needs to exist a fine-grained characterization of the user's experience in terms of satisfaction or acceptability, i.e. a quantification of QoE. Conversely, the more generic such a measure attempts to be, the more complex it becomes.

\section{A. The BetterX Project}

This article addresses the challenge of measuring MWQoE via BetterX, an end-to-end mobile-to-cloud system (MCS), which has been proposed as an architecture in [4] and has now been partially developed for the purposes of this work. BetterX has been designed primarily as a system of continuous MWQoE evaluation, where the BetterX Android client delivers context data to the BetterX Cloud implementation for processing, with the calculated MWQoE metric being sent back into the mobile device to possibly trigger actions, i.e. automations, that would benefit the user. The primary purpose of implementing BetterX for this study is to capture the training dataset for our MWQoE model and deliver a proof-of-concept system to motivate future industry-wide adaptations of MWQoE.

The BetterX approach differs from current QoE literature since it uses real-world data collected from the BetterX Android App. The BetterX dataset is collected automatically, in a non-intrusive manner without any user intervention and questionnaires. Furthermore, BetterX uses a novel fusion of data sources such as context data and web metrics in order to identify a distinct profile for each user and align user context with web metrics to infer the user's web session intention. This is then aligned with the web metrics and inferred device and 
network context states to build the MWQoE Bayesian model which delivers the MWQoE metric as a utility of the MWQoE context state.

The contribution of this work is the novel MWQoE model, which uses the largest set of context-attributes ever assembled for QoE, and the MWQoE metric which rates user satisfiability on selected scenarios on a single scale.

\section{RELATED WORK}

\section{A. Defining $Q o E$}

QoE can be understood as the quality perceived by the user in using an application or service. Given that there is current literature dealing with the criteria for measuring QoE for specific scenarios, there is still no universally accepted definition actualized in order to produce a quantifiable metric in real-world settings. Nevertheless, it is accepted that the term $\mathrm{QoE}$ refers to the perception of the user, with regards to the quality of a particular service or network [5]. More specifically, QoE is defined as the overall acceptability of an application or service, as perceived subjectively by the end user [6].

Currently, QoE research must consider the recent paradigm shift from a network-centered system perspective, to a user-centric one. Consequently, the networkcentered focus on the QoS metric, as the standard quality concept, is becoming obsolete, and, quantification of perceived quality based on metrics such as throughput, delay, jitter etc., is no longer sufficient.

Although many definitions on QoE have been published [7][8][9], this study adopts the ITU-T definition [10] and adapts it for the mobile web with the inclusion of web usage metrics. The ITU-T definition defines QoE as the overall acceptability of an application service, as perceived subjectively by end-users. Also, QoE includes the complete end-to-end system effects (client, terminal, network, services infrastructure, etc.). The overall acceptability may be influenced by a series of factors including user expectations, the usage context, the device usability and the user's personality.

\section{B. Mobile Web QoE (MWQoE)}

MWQoE measures the experience of the end user while using the web via a mobile device. There is an increasing need for wireless web access from a wide range of mobile devices such as smartphones, tablets and notebooks [11]. Such devices are characterized by limited keyboards, small screens, occasional low bandwidth connections, and, limited memory, amongst other constraints. Because of these constraints, mobile devices could benefit from special considerations when accessing information over the Internet. In fact, there is a major issue with rendering web content on mobile devices [12], which leads to a low web browsing QoE of the end user.
The World Wide Web Consortium (W3C) defines adaptation as a process of selection, generation or modification that produces one or more units of perception in response to a single requested resource [14]. Hence, adaptation is defined as any automatic action that adapts the content and presentation to improve user interaction with mobile handheld devices. These systemic effects may include filtering unwanted information and only provide information relevant to the needs of users, as well as data pre-fetch, triggered by changes in the environment or atmosphere. Based on previous studies on the adaptation of mobile web applications, this study classifies existing adaptive approaches into three main categories: content selection, content personalization and adaptation of the presentation [15].

Moreover, given the subjective nature of $\mathrm{QoE}$, the latest literature fuses computing topics with human behaviour studies. QoE must consider well-known laws from the field of human perception, as QoE directly relates to it. It has been shown that the Weber-Fechner Law, from psychophysics considering waiting times as stimulus, explains some elements of QoE [9].

Finally, some of the latest Web QoE literature [8], focuses on web traffic analysis and on QoE evaluation methods which are non-intrusive and do not rely on the user's view of quality using Mean Opinion Score (MOS). However, most WQoE characterizations are either abstract or limited in the sense that they only involve a subset of the attributes which have been shown to affect QoE. Therefore, there is a need to evolve and enhance current WQoE definitions.

\section{The BetterX ApProACH}

For the purpose of constructing the MWQoE model, and adapting the QoE definition into a practical MWQoE model, a novel fusion of context states [16] is used to build a model of a Bayesian Network [17], linking into a MWQoE metric based on Utility Theory [18]. The MWQoE model is evaluated by a second data collection of actual user feedback from a lab experiment.

\section{A. Context Awareness}

The user's context in selected scenarios is used to support inferences of the user's mobile web browsing experience, for instance, user situations may be infered based on context spaces theory [16]. In fact, context is any information that can be used to characterize the situation of an entity, such as a person, place, or object that is considered relevant to the interaction between a user and an application, including the user and applications themselves [19]. Thus, context-awareness entails the use of primary context types such as location, identity, activity, time to characterize a particular entity [19].

For MWQoE, these primary context types are mapped into 6 distinct data domains: device, network, connection, web, sensor, and user. The selected context domains 
encompass a total of 65 attributes (Section III-C), to be used in order to interpret the situation in which a web session of a particular user has taken place, in a specific location, at a specific time.

The method of context reasoning and interpretation, as illustrated in [20], is used to infer these situations using a three-stage process. Firstly, the context data, which is captured by sensors (such as GPS for location context) is considered. Secondly, this work considers a semantic interpretation of a higher-level context, i.e. the situation, and, thirdly, context interpretation needs to consider any relationship(s) between the situations. The idea behind context reasoning and interpretation is to infer sensor perceptions, or interpret metrics given an understanding of the user's situation. For MWQoE, this context-aware method is used to generate User Profiles and User Web Session Intentions based on an observation of the attributes in the 6 data domains.

\section{B. Decision Theory}

The underlying theoretical framework for modelling MWQoE is Decision Theory, which combines probability theory and utility theory [18]. Given that MWQoE deals with the interpretation of user context and any interpretation cannot capture the whole truth about a specific situation, then the MWQoE approach needs to account for uncertainty in user scenarios. The MWQoE metric, by definition, is highly aligned with the definition of utility theory, such that every state has a degree of usefulness, or utility, to an agent, and that the agent will prefer states with higher utility [18]. Thus, the higher the utility the higher MWQoE metric for the user.

The MWQoE model uses each collected data attribute as a Bayesian Network node, clustering nodes together for each of the domains of context data collected. The relationships in the Bayesian network are coded based on findings from current literature [3], as well as findings from this study's data analysis using Nave Bayes models to identify relationships and define strengths of influence of attributes with each other [21]. Nave Bayes modeling uncovers hidden relationships of attributes, given a set of training data of observations of a specific entity. The relationships uncovered in this study are used as expert knowledge, and define the relationship between each context state.

\section{BetterX Attribute List}

The list of data attributes designed to be collected by the BetterX Android application covered 6 different domains: 1 Device, 2 Network, 3 Connection, 4 Web, 5 Sensor, and 6 User. It was a large dataset composed of 65 attributes aimed in capturing and understanding the user's physical context, the network and connection state of the mobile device, the capabilities of the device and more importantly the web activity. Given the non-intrusive nature of this study, the Data Collection
TABLE I

EXAMPLES OF CONTEXT ATTRIBUTES FROM THE 6 DATA CATEGORIES

\begin{tabular}{ccc}
\hline Device & Network & Connection \\
Timestamp & Link Speed & Connection \\
Apps Launches & Has Internet & Strength \\
Location & Signal Strength & Web \\
Battery Level & Sensor & Tab \\
Dev. id & GPS & Page Info \\
Screen Status & User & HTTP Request \\
Phone Events & Timezone/Location & HTTP Response \\
\hline
\end{tabular}

Attribute List, as shown in Table III-C, was designed to enable an accurate interpretation of the user's surrounding conditions when using the web from a mobile device. Context-states were aimed to be derived from the Data Collection Attribute List and to be examined alongside web usage metrics for understanding the user scenarios of each of the observed web sessions and evaluating the experience index (MWQoE metric) for each one of them.

It is important to note that the BetterX MWQoE model was designed to not depend on QoS metrics but directly consider them.

The Device attributes capture basic information about the device, its capabilities and screen size, as well as a list of applications running and basic phone actions such as phone calls, screen switch offs, etc. The Network attributes captured the state of the network as it was received from the network provider (either Mobile Network or Wi-Fi). The Connection attributes outlined the current and available connections of the device. The Web attributes captured all available web session HTTP metrics from the Application Layer such as the Content Load (the number of milliseconds for the content of the page to be loaded from the initial request) and Page Load (the number of millisecond for the page to be completely loaded; when the onLoad event is fired) as well as HTTP POST/GET request details [22]. The Web attributes were based on the HTTP Archive format (HAR) [23]. For retaining user anonymity in HTTP request and response data, content was not captured and values from the request and response parameters were stripped away. The Sensor attributes logged data for each of the sensors that were available on the mobile device. The User attributes were captured via a very basic questionnaire upon installation of the BetterX Android App.

\section{Inferring User Web Session Intent}

Certain collected attributes were intended to be used either to extract, classify or estimate further information about the user to produce an estimation of the Web Session User Intent, Fig. 1. The Web Session User Intent was designed to be inferred by examining the HTTP Response and Request details (which revealed further insights about the interest of the user for the 


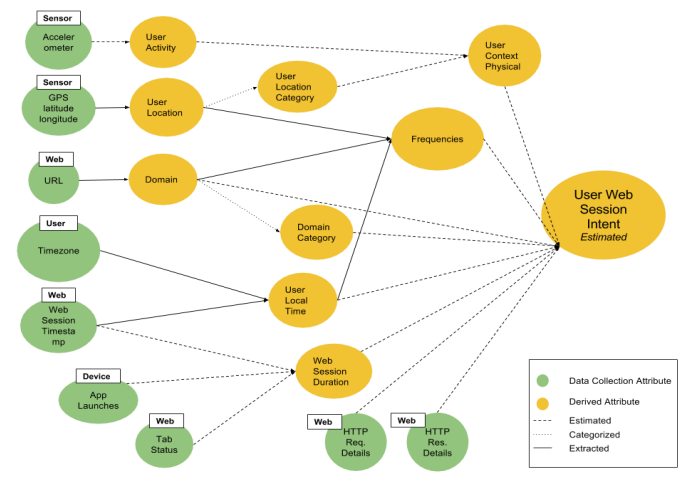

Fig. 1. Estimating User Web Session Intent

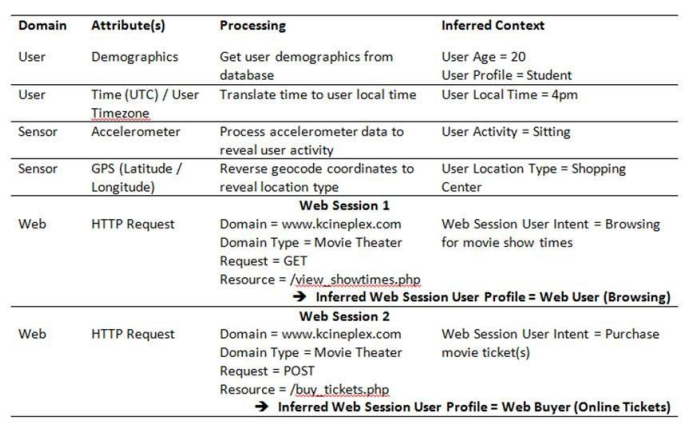

Fig. 2. Sample Data for User Intent \& User Profile

domain), the Web Session Duration, the User Local Time, the Domain and Domain Category, the User Physical Context (Activity, Location) and Frequencies (which revealed potential Time-Location-Domain relationships). The estimated Web Session User Intent revealed the Web Session User Profile (the classification of the type of the user). For example, the sample data in Fig. 2 below led to the following narrative about the observed user web sessions:

The user is a 20-year-old student (derived from Demographics) sitting (derived from Activity) in a shopping mall (derived from Physical Context) visiting the website of a nearby cinema (Domain, Domain Category) with the intent to purchase movie show time tickets (Frequencies, HTTP Request and Response).

Domains were designed to be extracted from URLs, Fig. 1, and categorized (Domain Category) using Amazon's Alexa API. The Web Session Duration was estimated by examining the Tab Status (Open/Closed) from which the web session was generated, the App Launches (if any apps were launched during the web session) and the Web Session Timestamp (the time which the web session was initiated).

For each of the 6 data domains collected, the study was designed to examine how, and to what extent, each attribute affected MWQoE. Device data was used to examine how MWQoE was affected by device characteristics, network data was used to examine how MWQoE changes based on network changes and so forth. The Data Collection Attribute List III-C combined with the Web Session User Intent, Fig. 1, provided a novel fusion of attributes.

\section{E. Inferring User Profiles}

The Web Session User Profiles were derived from the Web Session User Intent and the frequency of the Web Session User Intent within the observed time-frame of each distinct web session user. For example, if a user was observed working (the web session user intent) on crowdsourcing sites (domain type), then user was classified as worker for the web sessions that matched that same intent-domain-url part criteria. In a similar manner and after manually reviewing and analyzing all 2727 web sessions, a total of 4 distinct User Profiles was derived: 1 Worker, 2 Buyer, 3 Reader, and, 4 Web User (Default).

\section{THE BeTterX MWQOE MODEL AND METRIC}

\section{A. Timings Context}

The Timings Context State (TCS) forms a discrete representation of the amount of time it takes to make a web page available to the user from initial request to completion. TCS is generalized into 5 states (Excellent, Very Good, Good, Fair, Poor) closely resembling the Likert scale, which is used for MOS evaluation. The TCS model evaluates the time it takes for a webpage to be made available to the user based on the size of the request, the size of the response and the total time it took to fetch and display the contents of the web page.

The Total Size of the Request (TRTS) is the sum in bytes of the Body Size Request and the Header Size Request. The Total Size of the Response (TRES) is the sum in bytes of the Body Size Response and the Header Size Response. TRES is linked with the Send attribute since the time that is needed to send a request is proportionally related with the size of it. The same applies with the TRTS and the Receive attribute as the time it takes to receive a response is proportionally related to the size of the response. The Total HTTP Timings (THTTP) is the sum in milliseconds of all the Timing attributes (DNS, Connect, Send, Wait, Receive, Blocked). DNS measures the time it takes to resolve the host name. Connect measures the time required to create a TCP connection. Send is the time to send the HTTP request to the server. Wait is the time waiting for a response from the server. Blocked is the time spent waiting in queue for a network.

For TCS and the rest of the sub-models (NCS, DCS, ICS), 95\% Credible Intervals (CI) are used. The kmeans clustering algorithm is used for discretizing each attribute into a default of 10 context states, i.e. bins. 


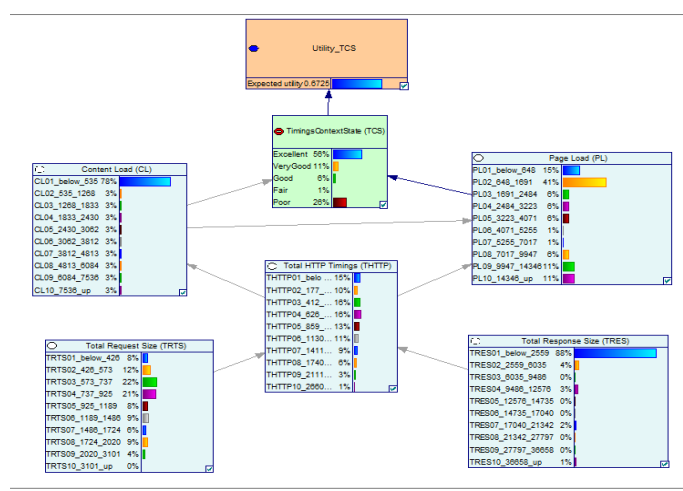

Fig. 3. TCS final Bayesian model

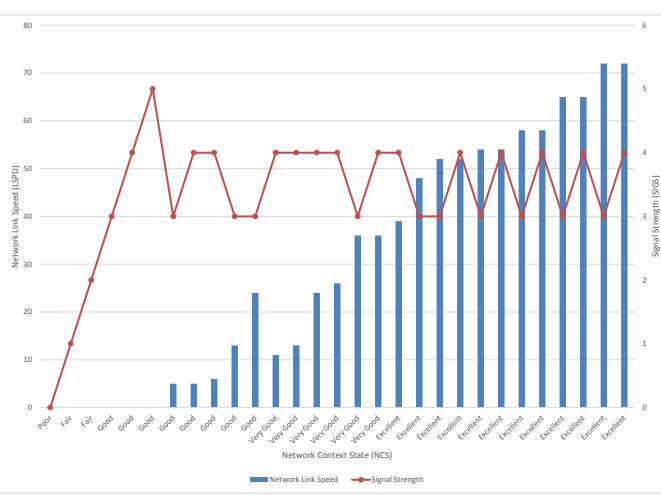

Fig. 4. NCS per signal strength and link speed
For example, the context-state of CL01 which is the 1st Bin of the Content Load attribute, is defined as Content Load of 0-535 milliseconds. The TCS States are linearly transformed and a Utility is assigned for each state. The Utility of TCS illustrates the timing of which the web page is made available to the user with the value 1 being Excellent and the value of 0 being Poor. The final TCS Bayesian model as it was coded in the GeNIe platform is shown in Fig. 3.

\section{B. Network Context}

The Network Context State (NCS) forms a discrete representation of the quality of the Network as it was received by the mobile device. Using the same methodology as the TCS model, The NCS model is generalized into 5 states (Excellent, Very Good, Good, Fair, Poor).

The three attributes that compose NCS are: 1 Internet, 2 Network Link Speed and 3 Signal Strength. The Internet (INT) attribute is of a binary format and shows if access to the Internet is available from the current connection. The Internet attribute is of a major strength of influence to NCS since a web page cannot be accessed without Internet. The Network Link Speed (LSPD) attribute measures the speed of network link in Mbps (Megabits per second) and the Signal Strength (SIGS) is a combination (union) of the Connection Strength (CSTR) and the Network Signal Strength (SIGL). CSTR measures the WiFi connection strength and SIGL measures the Mobile Network connection strength. The higher the signal strength and speed of the connection, the higher the NCS state.

The mapping between LSPD, SIGS and NCS reflects the predicate that SIGS and LSPD share the same strength of influence in NCS. Therefore, SIGS and LSPD are evenly distributed in NCS. Fig. 4 shows that when LSPD $=36 \mathrm{Mbps}$ and SIGS $=3$ then $\mathrm{NCS}=$ Very Good, whereas LSPD $=55$ and SIGS $=4$ gives $\mathrm{NCS}$ $=$ Excellent.

\section{Device Context}

The Device Context State (DCS) characterizes the availability of the device in regards to the amount of applications running on the device, the battery state of the device and the amount on telephony traffic on the device. DCS models the device usage frequency in regards to apps, phone and battery. Device usage has an inverse relationship with device availability, i.e. the available resources to use for web session downloading and content rendering depend on the amount of resources used for other tasks on the device such as applications running, the power available of the device and the actual telephony usage.

\section{Web Intent Importance Context}

The Web Intent Importance Context State (ICS) uses 4 attributes: The Location Type (LOC) from which the web session is generated, the Domain Type (DOMT) of the web session's URL, the User Type (UTYPE) or Web Session User Profile, as it has been outlined in BetterX User Profiles, and the Web Session User Intent (INTNT) which defines the intention of the user for initiating the web session. The Web Intent Importance Context State provides a characterization of the importance of the web session to the user. ICS is used in the MWQoE model based on the assumption that different types of requests hold different importance levels to users in specific scenarios. Once all the BetterX web sessions are coded with ICS levels and all the discrete classifications (INTNT, LOC, UTYPE, DOMT) are applied, then the resulting dataset is used to automatically generate the ICS Bayesian model using the Augmented Nave Bayes Approach.

\section{E. The Generated Model}

The final MWQoE model, as it is illustrated in Fig. 5 characterizes the experience of the mobile web user in regards to Timings Context (TCS), Device Context (DCS) and Web Intent Importance (ICS). TCS and DCS are fused together to derive the Web Immediacy State 


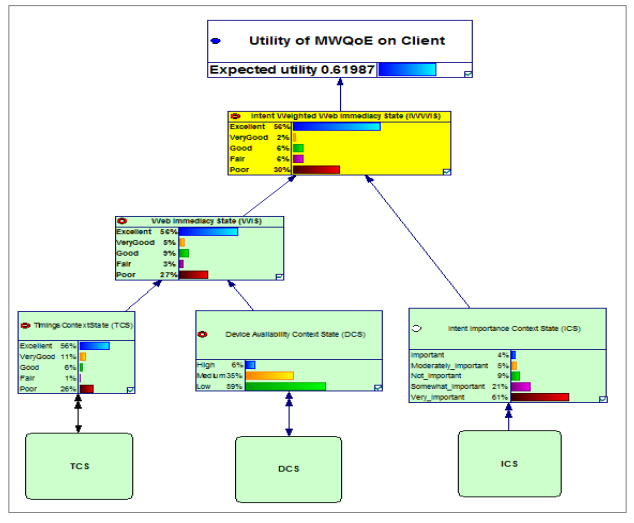

Fig. 5. Generated MWQoE model

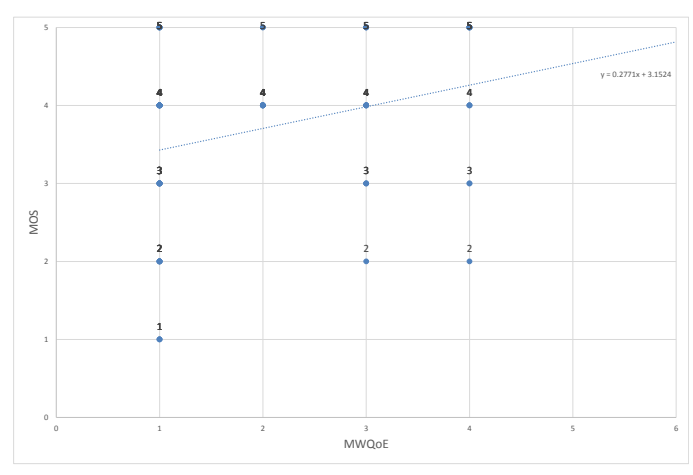

Fig. 6. Mapping MWQoE \& MOS relationship
(WIS). WIS represents the immediacy of the web page which is affected by the web timings (TCS) and the availability of the device (DCS); both attribute states which cause a web page to be either instantly available to the user (from initial request to a ready state) or have a noticeable delay in both content fetching and/or content rendering. Once WIS is derived, then it is combined with ICS (Intent Content State) to derive the Intent Weighted Web Immediacy State (IWWIS). IWWIS considers the immediacy of the page in regards to the derived Intent Importance of that web page to the user. The Utility of IWWIS is derived in a linear manner and is the final deliverable of the MWQoE model: the MWQoE metric. The MWQoE metric is linearly transformed on a scale from 0 to 1 as it was initially intended for this study.

The WIS state is predicated by the assumption that the better the web metrics (lower values) and the device availability, the better the web immediacy for the user and vice versa. The Page Load time (PL) can be affected by the processing power and memory available of the device (higher PL with slower machines), but, the processing power of the device cannot be affected by the Page Load time. The IWWIS state is composed by fusing together WIS and ICS. WIS is given a slightly stronger influence to IWWIS than ICS given the consideration that immediacy is more important to the user than the intention of the web session. Finally, the complete MWQoE Bayesian model is coded in the GeNIe platform, as shown in Fig. 5, which reveals that the mean MWQoE metric of all observed web sessions is 0.62 (Good), that $56 \%$ of all web sessions are found to have Excellent MWQoE, 2\% Very Good, 6\% Good, $6 \%$ Fair and $30 \%$ Poor.

\section{F. Model Evaluation via lab testing}

The comparison between the user MOS across all profiles closely compares with the MWQoE metric (Fig. 6). The comparison reveals a conservative MWQoE metric, one which provides either the same or a lower evaluation of the user's satisfiability in $93 \%$ of all comparisons. This feature works for the benefit of the user, as it has been intended for this project, since a lower metric can provide the basis for potential enhancements to the user. In addition, this comparison reveals that MWQoE is evaluated closely with the actual user averages since $66 \%$ of total observations are found to be either the same or close to the model (1 or 2 degrees difference). Furthermore, and given that this project is the first attempt to provide a unifying metric for MWQoE, the $28 \%$ of all observations which have been found to digress further from actual user opinion ( 3 and 4 degrees difference) together with the $6 \%$ where user opinion is lower than the metric are the basis for improvement for future work with a greater sample size.

The MOS vs MWQoE evaluations for all tasks are performed in the lab experiment across all profiles. The Context-States are generated for MWQoE based on the data collected from the smartphone and tablet in the lab experiment. The MOS for each task is compared with the MWQoE metric of the same task. Results show that $66 \%$ of all comparisons closely reflect user opinion, whereas the records highlighted with shades of orange $(28 \%)$ reveal a higher digress of the MWQoE metric from user opinion. The records highlighted with grey $(6 \%)$ indicate the observations where the metric gave a higher evaluation than the user.

The scatter plot (Fig. 6) shows the relationship between MWQoE and MOS. The generated trend line of $\mathrm{y}=0.2771 \mathrm{x}+3.1524$ reveals a positive gradient where the $y$ values are the discrete MOS ratings of the user and the $\mathrm{X}$-axis are the MWQoE evaluations generated by the lab experiment device data together with the TCS, DCS and ICS context-states. The positive gradient, evident in Fig. 6, shows that the higher the MWQoE prediction, the higher the user reported MOS. The offset from the origin of the line $y=0.2771 \mathrm{x}+3.1524$, crossing the $\mathrm{y}$ axis 3.1524 units above zero, shows that the MWQoE model is quite more conservative than the MOS ratings of the users. In fact only $6 \%$ of the total web session observations MOS was higher than MWQoE and in 
94\% the MWQoE model provided the same or a more conservative evaluation.

The conservatism of the MWQoE model is a significant result as it works for the benefit of the user since a lower system MWQoE prediction, in comparison with the actual user rating, provides the basis for potential enhancements to the device and subsequently an improvement to the user's MWQoE. Moreover, this finding reinforces literature on the Social Desirability Bias [24] in which users tend to answer more favourably and exaggerate ratings.

\section{CONCLUSION}

MWQoE can be considered the first practical usercentered attempt to measure Web QoE in specific mobile scenarios by using context-awareness and a non-intrusive approach. This study constructed MWQoE organically from live user data and has verified its validity in specific scenarios by benchmarking the MWQoE metric with user satisfiability ratings from a lab experiment.

The novelty of the MWQoE approach which fused together the User Web Session Intent with the Timings Context State and the Device Context State provided a practical novel Web QoE characterization. This was delivered by the custom built BetterX system, a novel MCC system, which exploited the sensing capabilities of modern mobile devices and the computational capabilities of the Cloud to provide a practical MWQoE model and metric in a non-intrusive manner, validated by labbased experiments (using users' subjective MOS). The proof-of-concept implementation of BetterX delivered by this work together with the complete end-to-end design and technical analysis provide a solution to both industry and academia for measuring, predicting and enhancing Web QoE for the benefit of both the online provider and the mobile user.

\section{ACKNOWLEDGMENT}

The authors would like to thank Amazon Web Services for supporting this work through an AWS Education Research Grant as well as with an AWS Cloud Credits for Research Program Grant.

\section{REFERENCES}

[1] S. Lanoue, What is UX Design? 15 User Experience Experts Weigh In, User Testing Blog, 2015. [Online]. Available: https://www.usertesting.com/blog/2015/09/16/what-is-ux-design15-user-experience-experts-weigh-in/. [Accessed: 01-Jan-2016]

[2] S. Egger, P. Reichl, T. Hosfeld, and R. Schatz, Time is bandwidth'? Narrowing the gap between subjective time perception and Quality of Experience IEEE Int. Conf. Commun., pp. 1325-1330, 2012.

[3] P. Fiedler, M.; Hossfeld, T.; Tran-Gia, A generic quantitative relationship between quality of experience and quality of service Network, IEEE, vol. 24, no. 2, pp. 36-41, 2010.

[4] E. Allayiotis and J. Antoniou, Technical considerations towards mobile user QoE enhancement via Cloud interaction in IWCMC 2014, 2014.

[5] Nokia Corporation, Quality of Experience (QoE) of mobile services: Can it be measured and improved?, 2004.
[6] ITU-T, Quality of service and performance - Generic and userrelated aspects, 2005.

[7] K. De Moor, W. Joseph, I. Ketyk, E. Tanghe, T. Deryckere, L. Martens, and L. De Marez, Linking users' subjective QoE evaluation to signal strength in an IEEE $802.11 \mathrm{~b} / \mathrm{g}$ wireless LAN environment, EURASIP J. Wirel. Commun. Netw., vol. 2010, p. 6, 2010.

[8] R. Schatz, T. Hofeld, L. Janowski, and S. Egger, From packets to people: quality of experience as a new measurement challenge, in Data traffic monitoring and analysis, Springer, 2013, pp. 219-263.

[9] P. Reichl, S. Egger, S. Member, R. Schatz, and A. D'Alconzo, "The Logarithmic Nature of QoE and the Role of the WeberFechner Law in QoE Assessment," IEEE ICC 2010, 2010.

[10] International Telecommunication Union, Vocabulary and effects of transmission parameters on customer opinion of transmission quality, amendment 2 P.10/G.100, 2006.

[11] E. Cecchet, R. Sims, X. He, and P. Shenoy, "mBenchLab: Measuring QoE of Web applications using mobile devices," in 2013 IEEE/ACM 21st International Symposium on Quality of Service (IWQoS), 2013, pp. 1-10.

[12] M. H. Zadeh, D. Wang, and E. Kubica, Human Factors for Designing a Haptic Interface for Interaction with a Virtual Environment, in Haptic, Audio and Visual Environments and Games, 2007. HAVE 2007. IEEE International Workshop on, 2007, pp. 21-26.

[13] R. Schatz, S. Egger, and A. Platzer, Poor, Good Enough or Even Better? Bridging the Gap between Acceptability and QoE of Mobile Broadband Data Services, 2011 IEEE Int. Conf. Commun., pp. 1-6, Jun. 2011.

[14] W3C, Glossary of Terms for Device Independence, 2005. [Online]. Available: https://www.w3.org/TR/di-gloss/.

[15] Y. Gong, F. Yang, L. Huang, and S. Su, Model-Based Approach to Measuring Quality of Experience, in 2009 First International Conference on Emerging Network Intelligence, 2009, pp. 29-32

[16] A. Padovitz, S. W. Loke, A. Zaslavsky, B. Burg, and C. Bartolini, An approach to data fusion for context awareness, Model. Using Context, pp. 353-367, 2005.

[17] D. Barber, Bayesian Reasoning and Machine Learning, Mach. Learn., p. 646, 2011.

[18] S. J. Russell and P. Norvig, Artificial Intelligence: A Modern Approach, vol. 9, no. 2. 1995.

[19] A. K. Dey, Understanding and Using Context, Pers. Ubiquitous Comput., vol. 5, no. 1, pp. 4-7, Feb. 2001.

[20] C. Bettini, O. Brdiczka, K. Henricksen, J. Indulska, D. Nicklas, A. Ranganathan, and D. Riboni, "A survey of context modelling and reasoning techniques," Pervasive Mob. Comput., vol. 6, no. 2, pp. 161-180, Apr. 2010.

[21] D. D. Lewis, "Naive (Bayes) at forty: The independence assumption in information retrieval," in Machine Learning: ECML98: 10th European Conference on Machine Learning Chemnitz, Germany, April 21-23, 1998 Proceedings, C. Ndellec and C. Rouveirol, Eds. Berlin, Heidelberg: Springer Berlin Heidelberg, 1998, pp. 4-15

[22] R. Fielding, J. Gettys, J. Mogul, H. Frystyk, L. Masinter, P. Leach, and T. Berners-Lee, "Hypertext transfer protocolHTTP/1.1," techreport, 1999.

[23] J. Odvarko, A. Jain, and A. Davies, "HTTP Archive (HAR) format," W3C Draft, 2012.

[24] C. Nancarrow, Brace, I. and Wright, L. T., Tell me Lies, Tell me Sweet Little Lies: Dealing with Socially Desirable Responses in Market Research, The Marketing Review, 2(1), pp. 5569, 2001. 